\title{
Semi-Lagrangian Scale Selective Two-Time-Level Scheme for Hydrostatic Atmospheric Model
}

\author{
Andrei Bourchtein ${ }^{1}$, Ludmila Bourchtein ${ }^{1}$, and Maxim Naumov ${ }^{2}$ \\ ${ }^{1}$ Institute of Physics and Mathematics, Pelotas State University, Brazil \\ bursteinaterra.com.br \\ ${ }^{2}$ Department of Computer Sciences, Purdue University, USA \\ naumov@purdue.edu
}

\begin{abstract}
A semi-Lagrangian scale selective finite difference scheme for hydrostatic atmospheric model is developed. The principal characteristics of the scheme are solution of the trajectory equations for advection, explicit first order approximation of physically insignificant adjustment terms and implicit time splitting discretization of the principal physical modes. This approach allows the use of large time steps, keeps practically the second order of accuracy and requires at each time step the amount of calculations proportional to the number of spatial grid points. The performed numerical experiments show computational efficiency of the proposed scheme and accuracy of the predicted atmospheric fields.
\end{abstract}

\section{Introduction}

Atmosphere parameters have a wide spectrum of spatial and temporal variations. Accordingly, the mathematical models of atmosphere (Euler or Navier-Stokes equations) contain solutions of different space and time scales. Analysis of the linearized equations reveals three principal types of atmospheric waves: acoustic, gravitational and inertial waves. Studying certain physical phenomenon one can try to filter the secondary effects still keeping all essential characteristics of the principal part. Analysis of the atmospheric data shows that weather systems are essentially defined by inertial motions while acoustic waves are practically neglectable. For large scale atmospheric dynamics the hydrostatic hypothesis is usually applied to filter out acoustic waves and simplify the governing equations. It has been proved to be effective simplification producing high quality atmospheric fields at reduced computational cost. Further attempts of simplification (Boussinesq, barotropic, quasigeostrophic, etc. approximations) have not been succeeded in keeping the same level of the forecast accuracy.

Although gravity waves seems to be analytically inseparable from inertial ones and their contribution to weather systems is not neglectable, one can try to divide the entire spectrum of these waves in more relevant and insignificant parts by using appropriate numerical methods. This approach can be practically implemented if vertical decoupling takes place. In fact, expansion of the atmospheric fields by vertical normal modes reveals high heterogeneity in the distribution of available energy: few greatest vertical modes are responsible for about ninety percent of total 
energy. This way, the general variability can be well predicted if the first vertical modes are approximated accurately while others are resolved more coarsely. Moreover, insignificant gravity waves contained in the smallest vertical modes can be approximated with even lower accuracy.

In this study, a scale separation is applied in the context of semi-Lagrangian semiimplicit (SLSI) method, which is currently the most efficient approach in numerical weather prediction and atmospheric modeling $[8,11,15,16]$. In this method the advective part is represented by equations of the trajectories of fluid particles, nonlinear terms are approximated explicitly and linear gravity waves implicitly along the above trajectories. At each time step the implicit terms require solution of 3D elliptic problem. Vertical decoupling transforms this problem into a set of 2D Helmholtz equations and eliminates necessity for implicit approximation of the insignificant vertical modes, but solution of the remaining elliptic problems is still expensive part of computations [4,7]. To overcome this difficulty, a time splitting method is applied to factorize each remaining 2D Helmholtz equation in a set of $1 \mathrm{D}$ problems, which are solved very effectively by direct Gelfand-Thomas algorithm. In order to reduce the splitting errors, which become great when time step exceeds the Courant-Friedrichs-Lewy (CFL) condition with respect to advection, the modified splitting proposed by Douglas et al. is applied $[6,9,10,17]$. This way, joining different numerical techniques we are able to construct computationally efficient and accurate SLSI model for the hydrostatic equations of the atmosphere.

\section{Primitive Equations}

Using time coordinate $t$, horizontal cartesian coordinates $x, y$ and vertical coordinate $\sigma=p / p_{s}$, the governing equations of the hydrostatic atmosphere can be written as follows [11]:

$$
\begin{gathered}
\frac{d u}{d t}=f v-G_{x}+N_{u}, \frac{d v}{d t}=-f u-G_{y}+N_{v}, \\
G_{\ln \sigma}=-R T, \\
\frac{d P}{d t}=-D-\dot{\sigma}_{\sigma}, \frac{d T}{d t}=\frac{R T_{0}}{c_{p}} \cdot\left(\frac{d P}{d t}+\frac{\dot{\sigma}}{\sigma}\right)+N_{T} .
\end{gathered}
$$

Here $u, v, \dot{\sigma}, G, P, T$ are unknown functions, namely, $u$ and $v$ are the horizontal velocity components, $\dot{\sigma}$ is the vertical velocity component, $D=u_{x}+v_{y}$ is the horizontal divergence, $P=\ln p_{s}, p$ and $p_{s}$ are the pressure and surface pressure respectively, $T$ is the temperature, $G=\Phi+R T_{0} P, \Phi=g z$ is the geopotential, $z$ is the height, $T_{0}=$ const is the reference temperature profile.

The nonlinear terms $N_{u}, N_{v}, N_{T}$ are expressed in the form

$$
N_{u}=-R\left(T-T_{0}\right) P_{x}, N_{v}=-R\left(T-T_{0}\right) P_{y}, N_{T}=-\frac{R\left(T-T_{0}\right)}{c_{p}}\left(\frac{\dot{\sigma}}{\sigma}-D-\dot{\sigma}_{\sigma}\right) .
$$


The individual $3 \mathrm{D}$ derivative is

$$
\frac{d \varphi}{d t}=\varphi_{t}+u \varphi_{x}+v \varphi_{y}+\dot{\sigma} \varphi_{\sigma} \quad, \varphi=u, v, P, T
$$

and the following parameters are used: $f$ is the Coriolis parameter, $g$ is the gravitational acceleration, $R$ is the gas constant of dry air, $c_{p}$ is the specific heat at constant pressure. Hereinafter the subscripts $t, x, y, \sigma$ denote the partial derivatives.

\section{Semi-Lagrangian Scale Selective Algorithm}

The developed algorithm follows the general outline of the two-time-level SLSI method $[11,15,16]$ with modifications imposed by application of vertical decoupling and horizontal splitting. First, the backward in time trajectory equations are solved:

$$
\frac{d \mathbf{r}}{d t}=\mathbf{V}, \mathbf{r}=(x, y, \sigma), \mathbf{V}=(u, v, \dot{\sigma}), t \in\left[t_{n}, t_{n+1}\right] ; \mathbf{r}\left(t_{n+1}\right)=\mathbf{r}^{a}
$$

Here $\mathbf{r}^{a}$ are given coordinates of the arrival points chosen to be the grid points. One standard procedure for finding the departure points $\mathbf{r}\left(t_{n}\right)=\mathbf{r}^{d}$ is solving (4) by the fixed point iterations $[11,15]$

$$
\Delta \mathbf{r}(s+1)=\tau \mathbf{V}^{n+1 / 2}(\mathbf{r}(s)-\Delta \mathbf{r}(s) / 2), \Delta \mathbf{r}(s)=\mathbf{r}^{a}-\mathbf{r}(s),
$$

with velocity defined by the extrapolation formula at the intermediate time level $t_{n+1 / 2}=(n+1 / 2) \tau: \mathbf{V}^{n+1 / 2}=\left(3 \mathbf{V}^{n}-\mathbf{V}^{n-1}\right) / 2$. Here $s$ is the iteration number and $\tau$ is the time step. If the iteration convergence condition [14]

$$
\tau \leq \frac{2}{3 V_{d}}, \quad V_{d}=\max \left(\left|u_{x}\right|,\left|u_{y}\right|,\left|u_{\sigma}\right|,\left|v_{x}\right|,\left|v_{y}\right|,\left|v_{\sigma}\right|,\left|\dot{\sigma}_{x}\right|,\left|\dot{\sigma}_{y}\right|,\left|\dot{\sigma}_{\sigma}\right|\right)
$$

is satisfied, then trilinear spatial interpolation of the velocity components to the trajectory points ensures finding the departure points with the second order of accuracy $[13,15,16]$. Using the maximum values of the wind component variations $V_{d} \approx 1.5 \cdot 10^{-4} \mathrm{~s}^{-1}$, the maximum allowable time step obtained from (6) is $\tau \approx 70 \mathrm{~min}$.

The second stage consists of semi-Lagrangian forward-backward approximation of the prognostic equations (1),(3):

$$
\begin{aligned}
& \frac{\hat{u}^{n+1}-u^{n}}{\tau}=f \hat{v}^{n+1}-\hat{G}_{x}^{n+1}+N_{u}^{n+1 / 2}, \frac{\hat{v}^{n+1}-v^{n}}{\tau}=-f u^{n}-\hat{G}_{y}^{n+1}+N_{v}^{n+1 / 2}, \\
& \frac{\hat{P}^{n+1}-P^{n}}{\tau}=-D^{n}-\dot{\sigma}_{\sigma}^{n}, \frac{\hat{T}^{n+1}-T^{n}}{\tau}=\frac{R T_{0}}{c_{p}}\left(\frac{\hat{P}^{n+1}-P^{n}}{\tau}+\frac{\dot{\sigma}^{n}}{\sigma}\right)+N_{T}^{n+1 / 2} .
\end{aligned}
$$


The nonlinear terms are found by extrapolation at the intermediate time level $t_{n+1 / 2}$ :

$$
N^{n+1 / 2}=\frac{N^{n+1 / 2}+N^{n+1 / 2}}{2}=\frac{1}{2}\left(\frac{3 N^{n}-N^{n-1}}{2}+\frac{3 N^{n}-N^{n-1}}{2}\right), N=N_{u}, N_{v}, N_{T} .
$$

The superscripts $n-1, n$ and $n+1$ denote the values at the past $t_{n-1}$, current $t_{n}$ and new $t_{n+1}$ time levels along the trajectory of air particles, that is,

$$
\varphi^{n+1}=\varphi\left(t_{n+1}, \mathbf{r}\left(t_{n+1}\right)\right), \varphi^{n}=\varphi\left(t_{n}, \mathbf{r}\left(t_{n}\right)\right), \varphi^{n-1}=\varphi\left(t_{n-1}, \mathbf{r}\left(t_{n-1}\right)\right), \varphi=u, v, G, P, T, D, \dot{\sigma} .
$$

Formulas (7),(8) can be solved in simple explicit way, but they have only the first order of accuracy and very restrictive CFL condition of stability:

$$
\tau \leq \sqrt{2} h_{g} / c_{g},
$$

where $h_{g}$ is a mesh size of spatial grid used for the gravity terms (that is, for the pressure gradient and divergence) and $c_{g} \approx 350 \mathrm{~m} / \mathrm{s}$ is the maximum velocity of the gravity waves in the primitive system. On the spatial grid $C$ with the main mesh size $h=50 \mathrm{~km}$, the minimum gravity mesh size is $h_{g}=h / 2=25 \mathrm{~km}$ [11]. Then the maximum allowable time step is about $100 \mathrm{sec}$, which is very small as compared with accuracy requirements. If the coarser mesh size of $75 \mathrm{~km}$ is used for approximation of the pressure gradient and divergence, then $\tau \approx 5 \mathrm{~min}$, which is still small.

The third stage consists of vertical decoupling and formulation of equations for implicit and more accurate approximation of the principal vertical modes. To this end, the equations for corrections to preliminary values are considered:

$$
\begin{gathered}
\frac{\delta u}{\tau}=f \frac{\delta v-\delta \hat{v}}{2}-\frac{\delta G_{x}-\delta \hat{G}_{x}}{2}, \frac{\delta v}{\tau}=-f \frac{\delta u+\delta \hat{u}}{2}-\frac{\delta G_{y}-\delta \hat{G}_{y}}{2} \\
\frac{\delta P}{\tau}=-\frac{\delta D+\delta \hat{D}}{2}-\frac{\delta \dot{\sigma}_{\sigma}+\delta \hat{\dot{\sigma}}_{\sigma}}{2}, \frac{\delta T}{\tau}=\frac{R T_{0}}{c_{p}}\left(\frac{\delta P}{\tau}+\frac{\delta \dot{\sigma}+\delta \hat{\dot{\sigma}}}{2 \sigma}\right) .
\end{gathered}
$$

Here $\delta \hat{\varphi}=\hat{\varphi}^{n+1}-\varphi^{n}$, and unknown functions found by the formulas

$$
\varphi^{n+1}=\hat{\varphi}^{n+1}+\delta \varphi, \varphi=u, v, G, P, T, D, \dot{\sigma}
$$

coincide with solution of usual two-time-level SLSI scheme described in $[12,16]$.

To avoid unnecessary corrections for insignificant vertical modes, the last system is vertically decoupled using the eigenvectors of the vertical structure matrix. To obtain these eigenvectors, the last two equations are simplified to the form

$$
\left(\sigma \frac{\delta T}{\tau}\right)_{\sigma}=-\frac{R T_{0}}{c_{p}} \frac{\delta D+\delta \hat{D}}{2}
$$


and, by using the hydrostatic equation (2), temperature $T$ is substituted by function $G$ :

$$
\left(\sigma \frac{\delta G_{\ln \sigma}}{\tau}\right)_{\sigma}=R T_{0} \frac{R}{c_{p}} \frac{\delta D+\delta \hat{D}}{2} .
$$

Equations (10), (12) form the closed system for three unknown functions. After vertical discretization on the Lorenz vertical $K$ level grid [2,3,5], the discrete analogues of equations (10), (12) can be written as follows:

$$
\begin{gathered}
\frac{\delta \mathbf{u}}{\tau}=f \frac{\delta \mathbf{v}-\delta \hat{\mathbf{v}}}{2}-\frac{\delta \mathbf{G}_{x}-\delta \hat{\mathbf{G}}_{x}}{2}, \frac{\delta \mathbf{v}}{\tau}=-f \frac{\delta \mathbf{u}+\delta \hat{\mathbf{u}}}{2}-\frac{\delta \mathbf{G}_{y}-\delta \hat{\mathbf{G}}_{y}}{2}, \\
\frac{\delta \mathbf{G}}{\tau}=-R T_{0} \mathbf{A} \frac{\delta \mathbf{D}+\delta \hat{\mathbf{D}}}{2},
\end{gathered}
$$

where $\mathbf{u}, \mathbf{v}, \mathbf{D}, \mathbf{G}$ are the vectors of order $K$ and $\mathbf{A}$ is $K \times K$ matrix of the vertical structure. The distribution of variables on the Lorenz vertical grid and some natural approximations to vertical operators can be found in $[2,3,5]$. Since discretization on this grid is more straightforward for keeping conservation properties of the primitive equations it seems to be the most popular vertical grid for hydrostatic models [2,5].

It was proved in [5] that the matrix $\mathbf{A}$ has the spectral decomposition $\mathbf{A}=\mathbf{S} \Lambda \mathbf{S}^{-1}$ with the positive eigenvalue matrix $\Lambda=\operatorname{diag}\left[\lambda_{1}, \ldots, \lambda_{K}\right]$ and the matrix of eigenvectors (that is, vertical normal modes) $\mathbf{S}$. Of course, all these transformations and calculations related to finding of the vertical structure matrix and its spectral decomposition are made only once before numerical forecasting.

Multiplying the equations of system (13) on the left by $\mathbf{S}^{-1}$, one obtains $K$ decoupled 2D systems

$$
\begin{gathered}
d u_{k}=\tau f \frac{d v_{k}-d \hat{v}_{k}}{2}-\tau \frac{d G_{k_{x}}-d \hat{G}_{k_{x}}}{2}, d v_{k}=-\tau f \frac{d u_{k}+d \hat{u}_{k}}{2}-\tau \frac{d G_{k y}-d \hat{G}_{k y}}{2}, \\
d G_{k}=-\tau c^{2} \frac{d D_{k}+d \hat{D}_{k}}{2} .
\end{gathered}
$$

Here $k=1, \ldots, K$ is the index of vertical mode, $c_{k}=\sqrt{R T_{0} \lambda_{k}}$ is the gravity wave speed of the $k$-th vertical mode and

$$
d \varphi=\mathbf{S}^{-1} \cdot \delta \varphi, d \hat{\varphi}=\mathbf{S}^{-1} \cdot \delta \hat{\varphi} \quad, \quad c_{k}^{2}=R T_{0} \lambda_{k},
$$

that is, $d \varphi_{k}$ are the coefficients of expansion of physical corrections $\delta \varphi$ by the vertical normal modes $\mathbf{s}_{k}$, which compile the matrix $\mathbf{S}$, and analogously for $d \hat{\varphi}_{k}$. The eigenvalues $\lambda_{k}$ (and $c_{k}$ ) are supposed to be numbered in decreasing order. From now on, we omit the subscript $k$, because it does not cause any ambiguity.

The last step of the algorithm is solution of (14) for the first principal $I$ modes (other modes remain without change) by applying a time splitting technique. Each system (14) for $i=1, \ldots, I$ is splitted into two subsystems solved successively for auxiliary corrections $d \widetilde{\varphi}$ and final corrections $d \bar{\varphi}$ : 


$$
d \tilde{u}=\tau f \frac{d \tilde{v}-d \hat{v}}{2}-\tau \frac{d \tilde{G}_{x}-d \hat{G}_{x}}{2}+h, d \tilde{v}=0, d \tilde{G}=-\tau c^{2} \frac{d \tilde{u}_{x}+d \hat{u}_{x}}{2},
$$

and

$$
d \bar{u}-d \tilde{u}=0, d \bar{v}-d \tilde{v}=-\tau f \frac{d \bar{u}+d \hat{u}}{2}-\tau \frac{d \bar{G}_{y}-d \hat{G}_{y}}{2}, d \bar{G}-d \tilde{G}=-\tau c^{2} \frac{d \bar{v}_{y}+d \hat{v}_{y}}{2} .
$$

Here $h$ is an additional modification term by Douglas et al. $[9,10]$. It can be shown that (16),(17) is the second order approximation to (14) and the splitting error can be reduced with no penalty on simplicity of algorithm by choosing function $h$ as follows:

$$
h=-\frac{\tau^{2} f^{2}}{4} d u^{*}-\frac{\tau^{2} f}{4}\left(d G^{*}-2 d \hat{G}\right)_{y}+\frac{\tau^{2} c^{2}}{4} d v^{*} x y, d \varphi^{*}=\varphi^{n}-\varphi^{n-1} .
$$

Each of the systems (16), (17) is transformed to $1 \mathrm{D}$ elliptic problem for $G$ corrections and the last is solved by simple Gelfand-Thomas algorithm. Found corrections are added to the values of the greatest modes and inverse vertical transformation returns the physical fields composed of the principal modes evaluated by semi-Lagrangian semi-implicit scheme and secondary modes calculated by equations (7),(8).

Applied scale separation allows to reduce the amount of computations at each time step and keep reasonably large time step. The linear stability analysis gives the approximate stability condition

$$
\tau \leq \sqrt{2} h_{g} / c_{I+1}
$$

for the explicit modes ( $c_{I+1}$ is the maximum gravity wave speed of the explicit modes) and the trajectory convergence restriction (6) for the implicit modes. Since gravity speeds decrease rapidly as $I$ increases, the explicit treatment of the smallest vertical modes does not cause strict limitation on the time step.

\section{Numerical Tests}

The described scheme was applied to 20-level hydrostatic model on horizontal grid with mesh size $h=50 \mathrm{~km}$. The first seven vertical modes with gravity wave speeds $c_{1}=343 \mathrm{~m} / \mathrm{s}, \quad c_{2}=203 \mathrm{~m} / \mathrm{s}, \quad c_{3}=122 \mathrm{~m} / \mathrm{s}, \quad c_{4}=78.1 \mathrm{~m} / \mathrm{s}, \quad c_{5}=54.3 \mathrm{~m} / \mathrm{s}$, $c_{6}=40.9 \mathrm{~m} / \mathrm{s}, c_{7}=32.6 \mathrm{~m} / \mathrm{s}$ were corrected and the remaining modes were treated explicitly with mesh size $h_{g}=75 \mathrm{~km}$ for the gravity wave terms. The greatest gravity speed of the explicit modes is $c_{8}=c_{I+1}=25.5 \mathrm{~m} / \mathrm{s}$ so that condition (18) allows to use time steps up to $60 \mathrm{~min}$, which is approximately equal to requirement (6) for the trajectory iterations. This way, the maximum time steps for traditional SLSI scheme (SLSIT) and described scale selective SLSI scheme (SLSIS) are practically coincide.

For evaluation of accuracy and computational efficiency of SLSIS scheme, its performance was compared with SLSIT scheme and with Eulerian leapfrog scheme 
(LF). The last is a simple rather popular explicit scheme tested in different models of the atmosphere. The integrations were carried out on horizontal domain of $5000 \times 5000 \mathrm{~km}^{2}$ centered at Porto Alegre city $\left(30^{0} \mathrm{~S}, 52^{0} \mathrm{~W}\right)$ and the initial and boundary conditions were obtained from objective analysis and global forecasts of the National Centers for Environmental Prediction (NCEP).

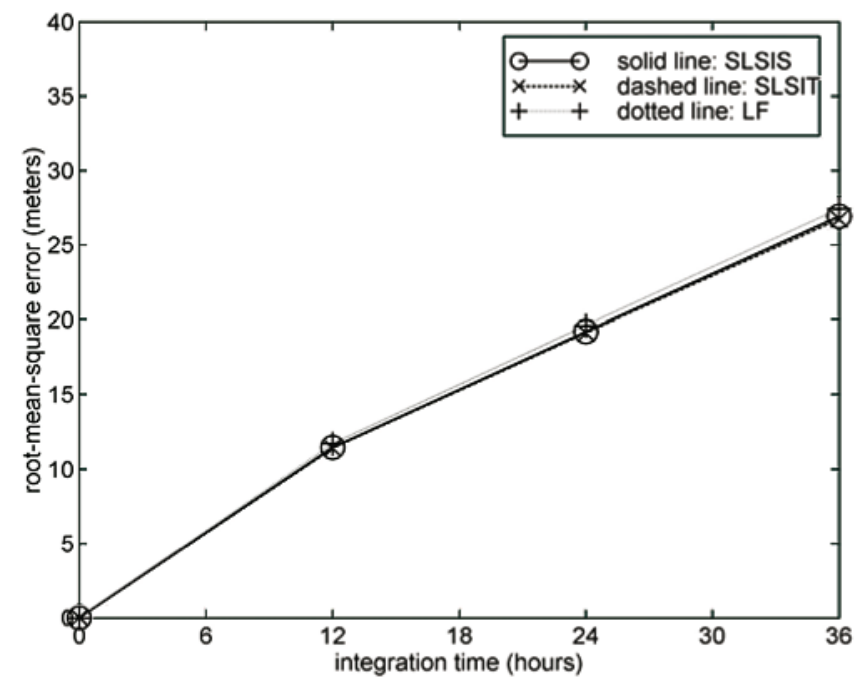

Fig. 1. Root-mean-square error of geopotential forecast at $500 \mathrm{hPa}$ pressure level

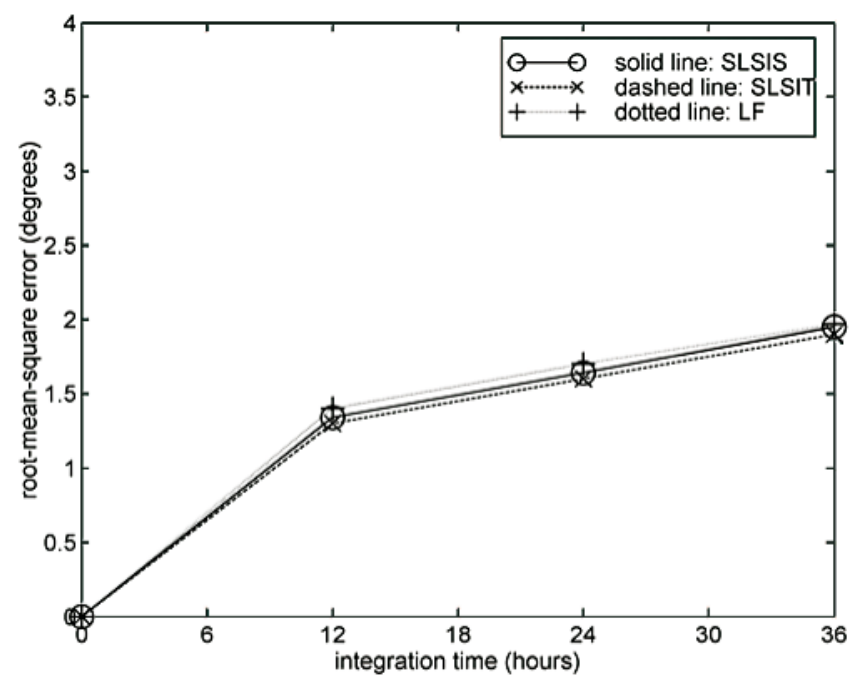

Fig. 2. Root-mean-square error of temperature forecast at $850 \mathrm{hPa}$ pressure level 
The 24-h forecasts were computed by using each of the above three schemes. The computational cost of one SLSIT forecast is about $18 \%$ of the LF computational time and the SLSIS scheme computation is about $42 \%$ faster than the SLSIT scheme. The root-mean-square differences between 24-h forecast and NCEP analysis are shown in Figs.1,2 for two elements: geopotential height at the 500hPa pressure level and temperature at the $850 \mathrm{hPa}$ pressure level. These characteristic elements are traditionally verified in the numerical weather prediction systems: the first reflects the dynamics of the middle atmosphere and the second is important for determination of humidity and cloud processes [1].

Another important skill measure for short-range forecasting is the correlation coefficient between predicted and observed tendency [1]. Evaluated for 12-h, 24-h and 36-h forecasts, this measure shows that the SLSIS and SLSIT forecasts are virtually coincide and the LF scheme has slightly lower accuracy, that is, it reveals relation between forecasts similar to that shown in Figs.1,2. Thus, performed evaluations confirm the validity of the applied method of scale separation and efficiency of the developed scheme.

Acknowledgements. This research was supported by Brazilian science foundation CNPq.

\section{References}

1. Anthes R.A., Kuo Y.H., Hsie E.Y., Low-Nam S., Bettge T.W.: Estimation of skill and uncertainty in regional numerical models. Q. J. R. Meteorol. Soc. 115 (1989) 763-806.

2. Arakawa A., Suarez M.J.: Vertical differencing of the primitive equations in sigma coordinates, Mon. Wea. Rev. 111 (1983) 34-45.

3. Bates J.R., Moorthi S., Higgins R.W.: A global multilevel atmospheric model using a vector semi-Lagrangian finite-difference scheme. Part I: Adiabatic formulation, Mon. Wea. Rev. 121 (1993) 244-263.

4. Bourchtein A.: Semi-Lagrangian semi-implicit space splitting regional baroclinic atmospheric model. Appl. Numer. Math. 41 (2002) 307-326.

5. Bourchtein A., Kadychnikov V.: Well-posedness of the initial value problem for vertically discretized hydrostatic equations. SIAM J. Num. An. 41 (2003) 195-207.

6. Browning G.L., Kreiss H.-O.: Splitting methods for problems with different timescales, Mon.Wea.Rev. 122 (1994) 2614-2622.

7. Burridge D.M., A split semi-implicit reformulation of the Bushby-Timpson 10 level model, Quart. J. Roy. Meteor. Soc. 101 (1975) 777-792.

8. Côté J., Gravel S., Methot A., Patoine A., Roch M., Staniforth A.: The operational CMCMRB global environmental multiscale (GEM) model. Part I: Design considerations and formulation, Mon. Wea. Rev. 126 (1998) 1373-1395.

9. Douglas J., Kim S., Improved accuracy for locally one-dimensional methods for parabolic equations, Mathematical Models and Methods in Applied Science 11 (2001) 1563-1579.

10. Douglas J., Kim S., Lim H., An improved alternating-direction method for a viscous wave equation, in: Z. Chen, R. Glowinski, Kaitai L. (Eds.), Current Trends in Scientific Computing, Contemporary Mathematics, 329 (2003) 99-104.

11. Durran D.: Numerical Methods for Wave Equations in Geophysical Fluid Dynamics. Springer, New York (1999). 
12. Hortal M.: The development and testing of a new two-time-level semi-Lagrangian scheme (SETTLS) in the ECMWF forecast model, Q.J.R.Met.Soc. 128 (2002) 1671-1687.

13. McDonald A.: Accuracy of multiply upstream, semi-Lagrangian advective schemes, Mon. Wea. Rev. 112 (1984) 1267-1275.

14. Pudykiewicz J., Benoit R., Staniforth A.: Preliminary results from a partial LRTAP model based on an existing meteorological forecast model. Atmos.-Ocean 23 (1985) 267-303.

15. Staniforth A., Côté J.: Semi-Lagrangian integration schemes for atmospheric models - A review. Mon. Wea. Rev. 119 (1991) 2206-2223.

16. Temperton C., Hortal M., Simmons A.J.: A two-time-level semi-Lagrangian global spectral model. Q. J. R. Meteorol. Soc. 127 (2001) 111-126.

17. Yakimiw E., Robert A.: Accuracy and stability analysis of a fully implicit scheme for the shallow water equations, Mon. Wea. Rev. 114 (1986) 240-244. 